\title{
Aplicación de metodología Pfafstetter para el estudio de identificación y análisis de micro cuencas de municipios de MAMCEPAZ
}

Claudia Nataly Mondragón R. Yessica Yamileth Sosa Reyes

\section{Resumen}

El artículo explica la aplicación de la metodología Pfafstetter para el estudio de identificación y análisis de microcuencas hidrográficas de los 8 municipios de la Mancomunidad MAMCEPAZ en el centro-occidente de Honduras. La aplicación de dicha metodología permitió la identificación de las unidades de drenaje basado en las características de la superficie del terreno las cuales poseen características territoriales particulares en los 8 municipios de estudio, se prevé que a partir de la identificación de las microcuencas, estas puedan ser gobernadas mediante la toma de decisiones basadas en un modelo en donde la gestión territorial tenga un papel protagónico para garantizar de esa forma el manejo sostenible de los recursos territoriales, incluidos el recurso suelo y agua.

Palabras Clave: Microcuenca, Territorio, Suelo, hidrografía, Pfafstetter.

\section{Abstract}

The Pfafstetter methodology has been applied within this case of study in order to identify and analyze a micro watershed that covers 8 municipality's in the middle west part of Honduras. Known as MAMCEPAZ. This exercise allows us to identify some draining units, based on the characteristics of the land's surface. We conclude that those characteristics could be governed making decisions based on a model in which the territorial management takes a protagonist role that guarantee a sustainable use to all the resources within the territory, especially water.

Keywords: micro watershed, Land, Territory, hydrography, Pfafstetter. 
Claudia Nataly Mondragón Rivera, (cnmondragon.rivera@gmail.com). Universidad Nacional Autónoma de Honduras, Facultad de Ciencias Espaciales, Departamento de Ciencia y Tecnologías de la Información Geográfica.

Yessica Yamileth Sosa Reyes, (yessica.sosa04@gmail.com), Universidad Nacional Autónoma de Honduras, Facultad de Ciencias Espaciales, Departamento de Ciencia y Tecnologías de la Información Geográfica.

Fecha de Recepción: 15 de febrero de 2017. Fecha de Aceptación: 24 de agosto de 2017. 


\section{INTRODUCCIÓN}

Esta investigación se constituyó como una necesidad de la Mancomunidad MAMCEPAZ en identificar de manera científica y técnica, las cuencas hidrográficas de los 8 municipios que conforman la Mancomunidad, mediante una metodología novedosa que permite identificar unidades de drenaje basado en la topología de la superficie del terreno. Esta metodología permite obtener las micro cuencas hidrológicamente ordenadas, proveyendo una única identificación a cada microcuenca, lo que dará eficiencia en el manejo de dígitos para cada una.

Después de haber identificado las microcuencas, se visibilizó con autoridades de la Mancomunidad, la necesidad de que la información vinculada a las unidades territoriales fuera actualizada y con una análisis territorial a detalle, para generar una herramienta de gestión territorial que esté acorde a las necesidades actuales en donde los territorios se vean afectados por amenazas diferenciadas tales como el gorgojo descortezador del pino que han venido a modificar el espacio territorial de manera reciente.

La información actualizada está vinculada a los datos del Censo de Población y Vivienda 2013 contrastada con el censo de población y Vivienda 2001, además de ello se establecieron los nombres para cada microcuenca, se determinó el uso predominante de suelo, se realizó un análisis cartográfico y una validación en campo en cada microcuenca. Cabe mencionar que estas actividades fueron desarrolladas con el apoyo de los miembros de la Unidades Ambientales y Unidades de Catastro de cada una de las municipalidades de la MAMCEPAZ.

Como resultado se obtuvo la propuesta de delimitación de 77 microcuencas en los 8 municipios de la mancomunidad, a las cuales además se realizó una propuesta de nombre, identificación de problemas y proyectos vinculados a los planes municipales de ordenamiento territorial, uso predominante de suelo y carga poblacional, aspectos que facilitarán la gestión territorial de las microcuencas.

\section{Marco Referencial}

El método de codificación de unidades hidrográficas fue creado en Brasil por Otto Pfafstetter en 1989 y difundido a partir de 1997 por Kristine Verdin a través del Servicio Geológico, de los Estados Unidos (USGS) en el programa Nacional del Medio Ambiente de las Naciones Unidas. En la actualidad va constituyéndose en un estándar de codificación de unidades hidrográficas. Es una metodología para asignar Identificadores (Ids) a unidades de drenaje basado en la topología de la 
superficie 0 área del terreno; asigna Ids a una unidad hidrográfica para relacionarla con las unidades hidrográficas que contiene y de las unidades hidrográficas con las que limita.

La delimitación de cuencas hidrográficas constituye un insumo esencial para llevar a cabo la planeación y la gestión de los recursos naturales bajo el enfoque de cuencas. Estas unidades territoriales están delimitadas por un parteaguas a partir del cual las aguas escurren y se concentran, pudiendo desembocar en el mar, en un cuerpo de agua interior o infiltrarse antes de encontrar algún cuerpo o superficie colector. (SUR, UICN, 2008)

En México se han hecho múltiples esfuerzos para elaborar divisiones hidrográficas, aunque pocas de ellas han seguido sólo criterios naturales, principalmente topográficos e hidrográficos. (INEGI-INE-CONAGUA, 2007)

Para el Perú, la elaboración de una base digital de cuencas hidrográficas es una necesidad, la utilización del método Pfafstetter para clasificación y subsecuente codificación de las cuencas hidrográficas es plenamente justificable, dadas a sus características únicas. De esta manera, permite que el código de cada unidad hidrográfica sea único dentro de un continente. (INSTITUTO NACIONAL DE RECURSOS NATURALES, 2005)

La elaboración de una base digital de cuencas hidrográficas para el área de estudio y para todo el Paraguay es una necesidad. La utilización del método Pfafstetter para clasificación y subsecuente codificación de las cuencas hidrográficas es plenamente justificable, dadas sus características únicas. (Antúnez, 2011)

El tema de Cuencas Hidrograficas en Nicaragua bajo la metodologia Pfafstetter, tiene como objetivo presentar una herramienta actualizada para la administración y gestión del agua según las cuencas hidrográficas a diferentes niveles y poder ayudar a las personas, familias y comunidades acompañadas de las Instituciones del Gobierno Nacional, Regional y Local en implementar la gestión integrada de los recursos hídricos y su planificación de cuencas como lo mandata la Ley General de Aguas Nacionales. (INETER, 2014)

De acuerdo con lo establecido al año 2022, el Instituto Nacional de Conservación y Desarrollo Forestal, Áreas Protegidas y Vida Silvestre (ICF) cuenta con el 100\% de las cuencas prioritarias, bajo régimen de manejo integral, a través de una efectiva armonización y coordinación interinstitucional y demás entes de la sociedad civil, producto de la implementación exitosa de los lineamientos estratégicos para el 
manejo de cuencas hidrográficas. Estableciéndose únicamente una delimitación integral de Cuencas y Sub cuencas. (ICF, 2010)

Bajo estos contextos y viendo la necesidad de una Planificación Territorial más eficiente en donde se incluyan todos los sectores productivos, se ve la importancia de incluir esta metodología en el área de estudio de la Mancomunidad de los municipios del Centro de la Paz, que en conjunto a su cartera de proyectos establecidos en el Plan de Ordenamiento Territorial visualizan las necesidades de cada comunidad inserta en cada una de las microcuencas resultantes.

\section{MÉTODO}

La metodología utilizada basada en un enfoque de un estudio mixto, a través de un diseño no experimental y con una temporalidad longitudinal, utilizando del Censo de Población y Vivienda del 2001 y 2013, identificado a través de giras de trabajo para la identificación de los datos territoriales, finalizando con el levantamiento de la información con utilización de sistemas de información geográfica para la validación de los datos encontrados. En el enfoque Cuantitativo se realizó con el proceso de recolección de información, identificando y codificando cada uno de los tipos de microcuencas de acuerdo con la metodología Pfafstetter y de los usos del suelo de cada una de ellas. En el desarrollo del enfoque cualitativo se realizaron reuniones focales con utilización de un instrumento de encuesta de trabajo, relacionado a temas de ambiente, Infraestructura y asentamiento humanos y la socioeconómicos, para la recolección de información de cada una de las instituciones del estado, internacionales, organizaciones locales y nacionales, así como la codificación, población, tipo de suelo, el nombramiento de cada microcuenca entre otros, relacionados a las comunidades que la comprenden y relacionados con la cartera de proyectos expuestos en el Plan de Ordenamiento Territorial.

\section{RESULTADOS}

A fin de mostrar los objetivos principales y específicos de este estudio la información pertinente a cada microcuenca identificada para la mancomunidad MAMCEPAZ, con utilización de técnicas cartográficas, información secundaria e información primaria, de modo que garantice una herramienta de gestión territorial actualizada y acorde a las necesidades de cada municipio que conforman la Mancomunidad, se desarrollaron en cinco faces descritas a continuación: 


\section{Fase I. Recopilación de la Información Secundaria}

Esta fase muestra los procesos de la preparación de recolección de la información, formando equipos de trabajo con miembros de las municipalidades de la MAMCEPAZ, siento este el primer acercamiento formal a la zona de estudio, se recopiló la información cartográfica, de contextos y de campo, relacionados al recurso agua, ambiental y socioeconómica, obteniéndose información cartográfica previa con utilización del Instrumento de la encuesta que acompañaba un mapa del área con sus zonas productivas de agua y límites municipales y en formato Shapefile (es un formato de archivo informático de datos espaciales), todos ellos siendo validadas con los entes encargados de las unidades ambientales de cada municipio, (Ver Figura 1) posteriormente se realizó verificación en campo, (Ver Figura 2), además la información fue complementada con información que se obtuvo del Sistema Nacional de Información Territorial SINIT.

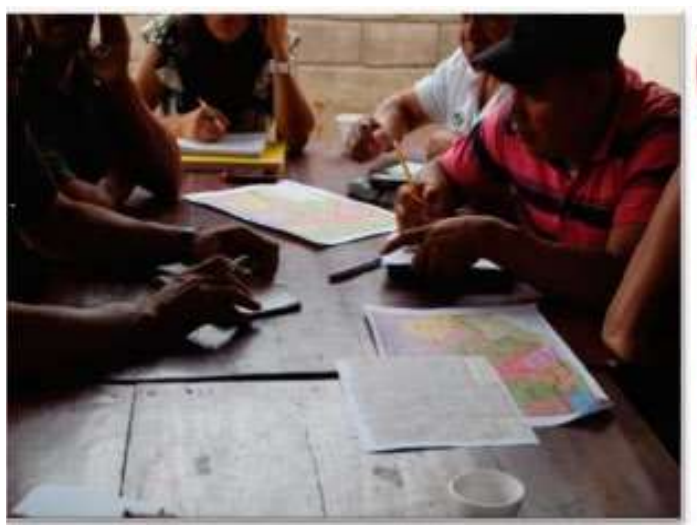

Figura 1. Equipo de trabajo con Técnicos municipales en el área ambiental de cada municipio de la MAMCEPAZ.

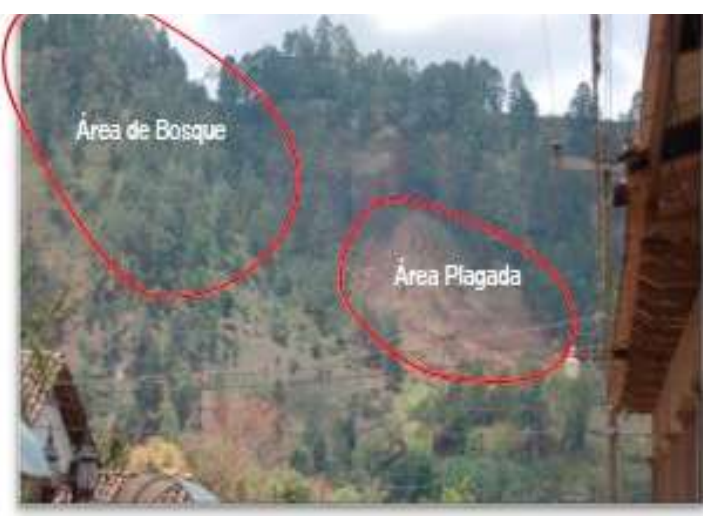

Figura 2. Validación de datos cartográficos. Foto: Yessica Sosa. 


\section{Fase II. Implementación de Metodología Pfafstetter}

Para iniciar el proceso de delimitación y codificación se utilizó el Manual de Delimitación y Codificación de Unidades Hidrográficas de Sudamérica, en donde se inicia determinando el curso del rio principal de la unidad que se va a codificar.

Se encontraron cuatro Ríos principales, ubicados en dos cuencas (Ver Tabla 1) (ver Figura 3).

Tabla 1. Ríos Principales y Cuencas.

\begin{tabular}{|l|l|l}
\hline No. & Nombre de Rio & Nombre de Cuenca \\
\hline $\mathbf{1}$ & Río Grande de Otoro & \\
\hline $\mathbf{2}$ & Río Humuya Alto & \\
\hline $\mathbf{3}$ & Río Selguapa & \\
\hline $\mathbf{4}$ & Rio El Venado & Cuenca Lempa \\
\hline
\end{tabular}

(Fuente: elaboración propia) 


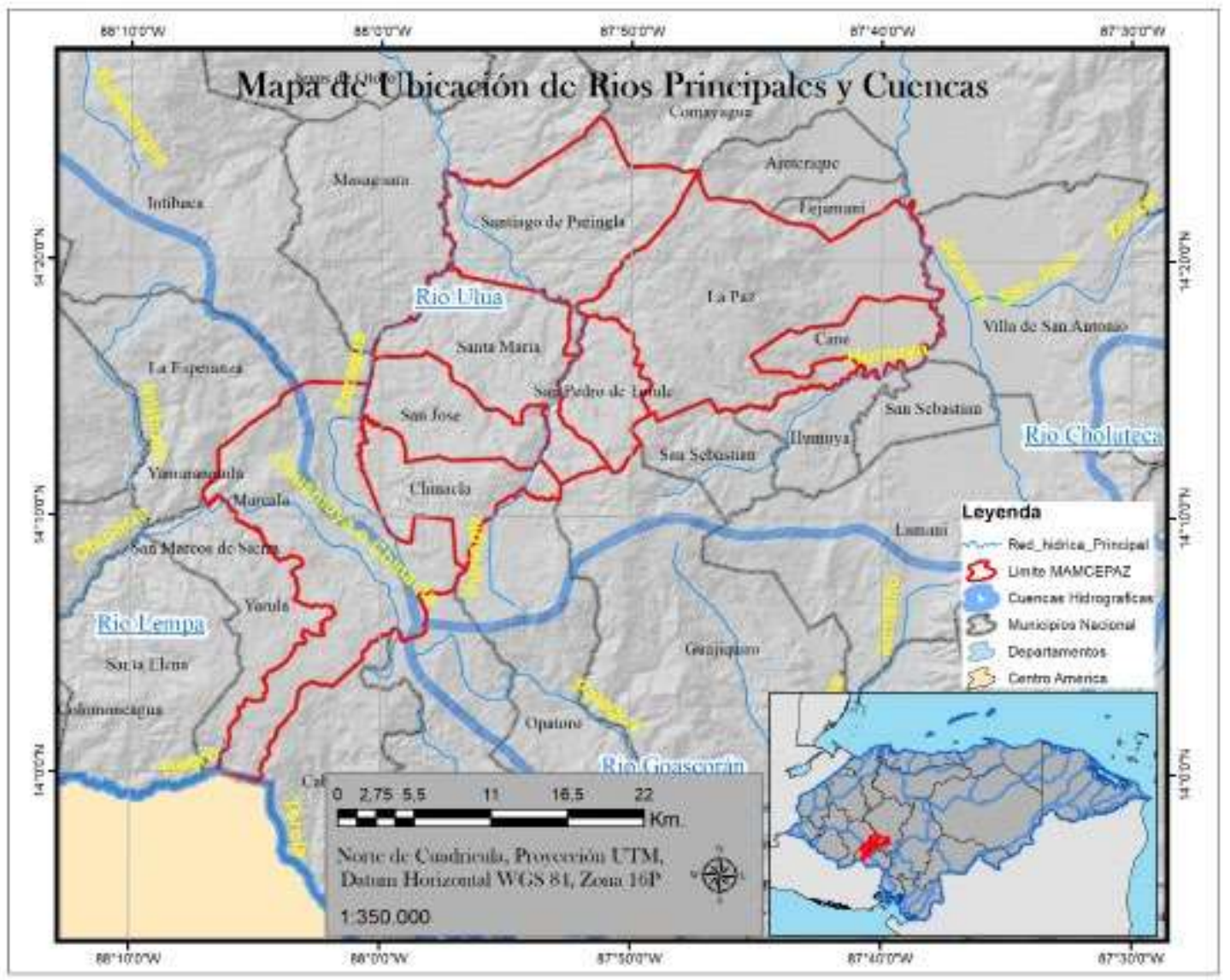

Figura 3. Ubicación de ríos principales y cuencas.

(Fuente: Elaboración propia).

Una vez obtenido el dato de figura (Shapefile) del curso del Rio Principal, se codificaron las cuatro unidades hidrográficas de tipo cuenca, que son las cuatro unidades de mayor área que confluyen en el rio principal, de acuerdo con la metodología Pfafstetter.

Para esto la Metodología Pfafstetter consideran tres tipos de unidades de drenaje:

Cuenca, es un área (unidad hidrográfica) que no recibe drenaje de ninguna otra área, pero si contribuye con flujo a otra unidad de drenaje.

Cuenca Intercuenca, es un área (unidad de drenaje) que recibe el drenaje de otra unidad que se ubica aguas arriba, mediante el curso del río principal, y permite el drenaje del flujo propio y del que ha ingresado a esta unidad hacia la unidad de drenaje que se ubica hacia aguas abajo. En tal sentido una unidad de drenaje tipo 
intercuenca es una unidad de drenaje o de tránsito del río principal al cual también aporta sus propios caudales.

Cuenca Interna, es un área de drenaje que no recibe flujo de agua de otra unidad ni contribuye con flujo de agua a otra unidad de drenaje o cuerpo de agua. (Ver Figura 4).

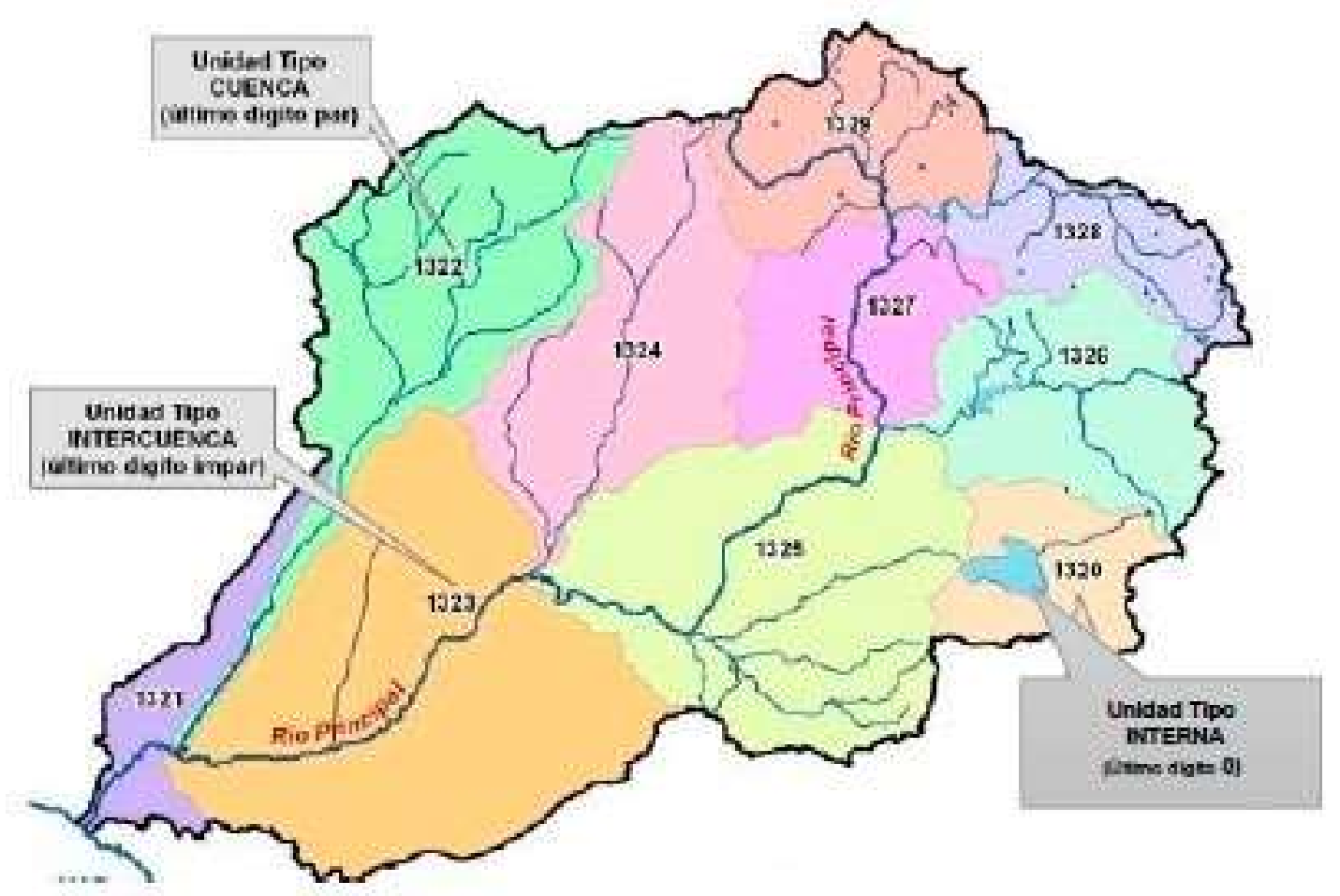

Figura 4. Tipo de Unidades Hidrográficas (SUR, U. (2008).

Las cuatro unidades tipo cuenca se codifican con los dígitos pares 2, 4, 6 y 8, desde aguas abajo hacia aguas arriba; es decir, desde la desembocadura hacia la naciente del río principal. Las otras áreas de drenaje se agrupan en unidades hidrográficas de tipo intercuenca, y se codifican, también desde aguas abajo (desde la confluencia) hacia aguas arriba, con los dígitos impares 1, 3, 5, 7 y 9.

De acuerdo con la metodología de delimitación y codificación de las unidades hidrográficas, el código 9 siempre resulta o se reserva para la unidad de drenaje de mayor tamaño de la parte superior de la cuenca o cabecera de cuenca la misma que generalmente contiene el origen del río cuya unidad de drenaje se está codificando. 
Cada una de las unidades de drenaje de tipo cuenca o intercuenca, delimitadas y codificadas en un determinado nivel (por ejemplo el primero) se pueden a su vez subdividir y codificar siguiendo exactamente el proceso antes descrito, de modo que por ejemplo la delimitación y codificación de la unidad de drenaje tipo cuenca de código 8 se subdivide, y se codifica en nueve unidades hidrográficas, cuatro de tipo cuenca códigos $82,84,86$ y 88 y cuatro de tipo intercuenca de códigos 81,83 , 85 y 87 así como la unidad hidrográfica de cabecera de código 89. (SUR, 2008) (Ver Figura 5).

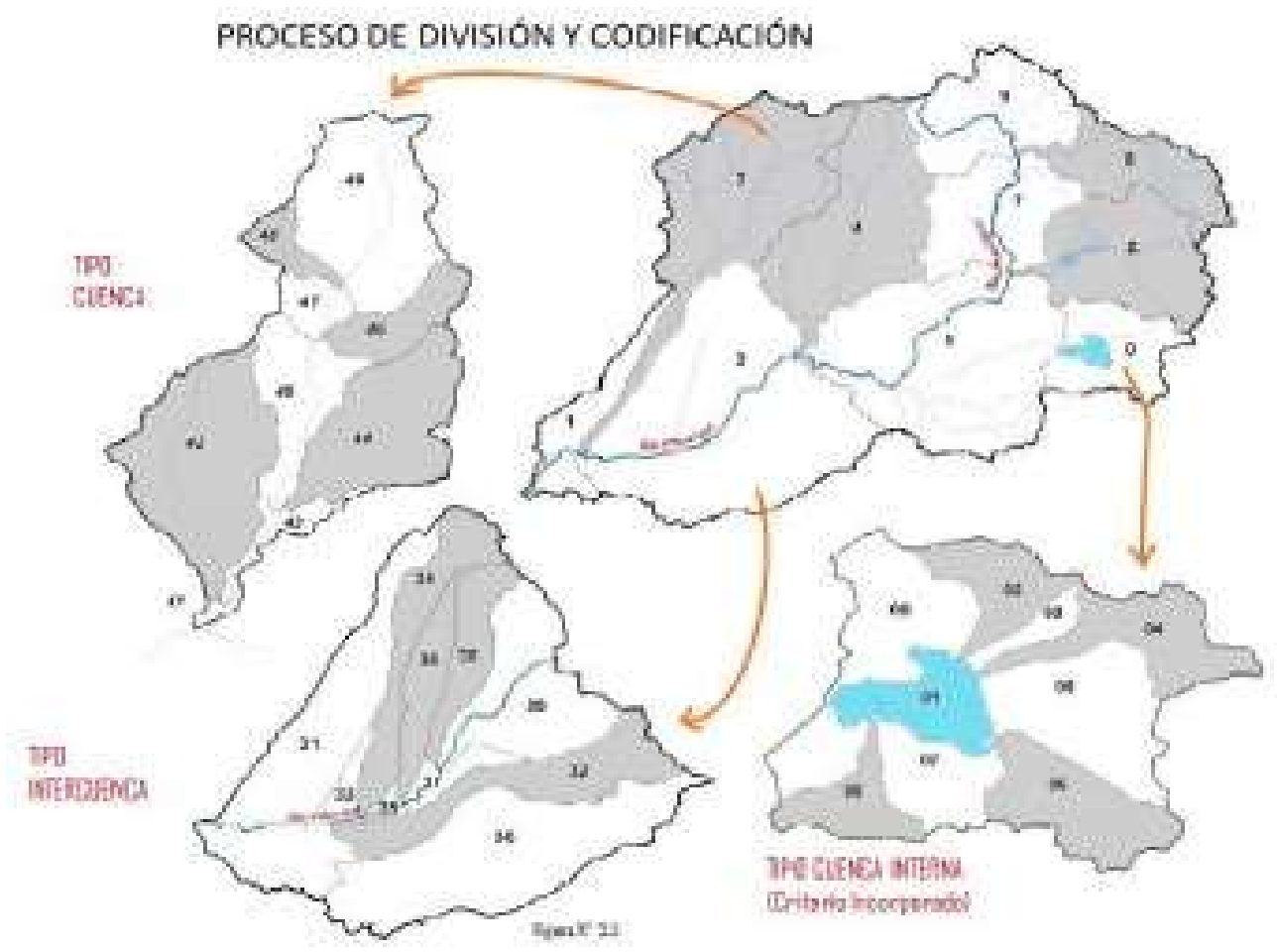

Figura 5. Proceso de División y Codificación según metodología Pfafstetter (SUR, U. (2008).

Para el caso de los municipios de MAMCEPAZ la distribución de los niveles de codificación queda de la siguiente manera:

Nivel 1. Para el desarrollo de esta segunda fase se utilizó información de base el estudio del mapa elaborado por el EROS DATA CENTER (Survey, 2016), tomando como base el Modelo de Elevación del Terreno GEOTOPO30, a nivel mundial, para la asignación del primer digito de la Intercuenca, en este sentido para la región que corresponde para Honduras sería el número 9. (Ver Figura 6). 


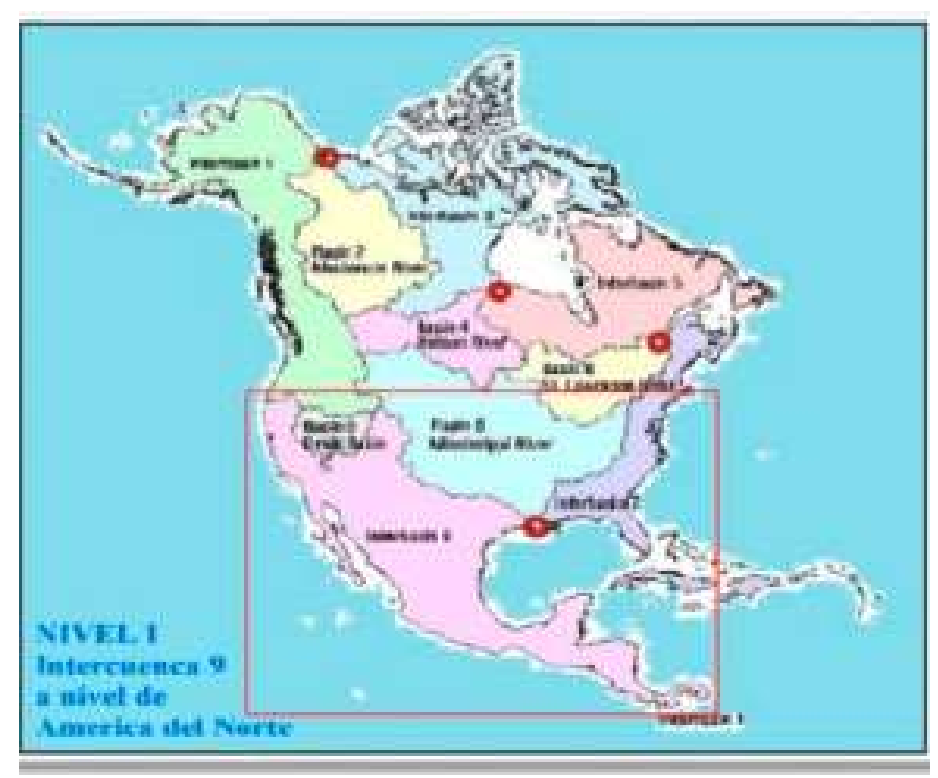

Figura 6. Nivel 1 Intercuencas 9. (Survey, 2016).

Nivel 2. En este mapa el inicio de la delimitación fue continental, separando en el caso de América en Norte y Sur América, Norte América se delimita para el nivel 1 en 9 cuencas, siendo la cuenca 9 la correspondiente a México y Centroamérica, la cual es una intercuenca. (Ver Figura 7.)

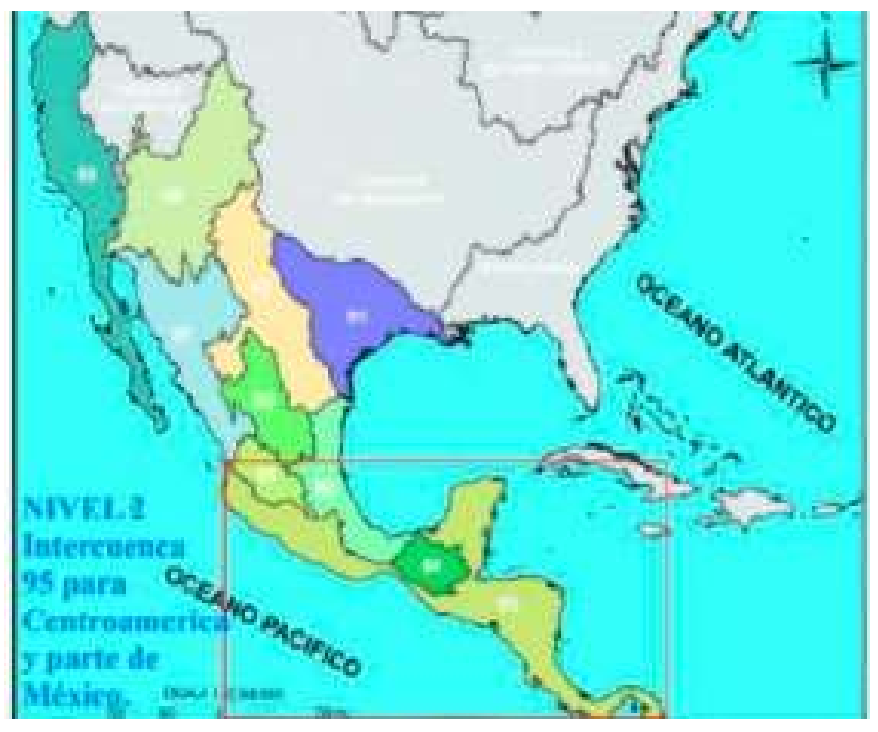

Figura 7. Nivel 2 Intercuenca 95. (Survey, 2016). 
Nivel 3. En el país no existe una clasificación a nivel 3 en adelante, por lo que será una gran oportunidad contar con el desarrollo de una delimitación, a los niveles $4,5,6,7$, u 8, permitiendo hacer estudios en unidades hidrológicas de cuenca, subcuenca y microcuenca.

Información utilizada en base a la Visión de País y Plan de Nación utilizando para el nivel 3 los números 1 y 5 . (Ver Figura 8).

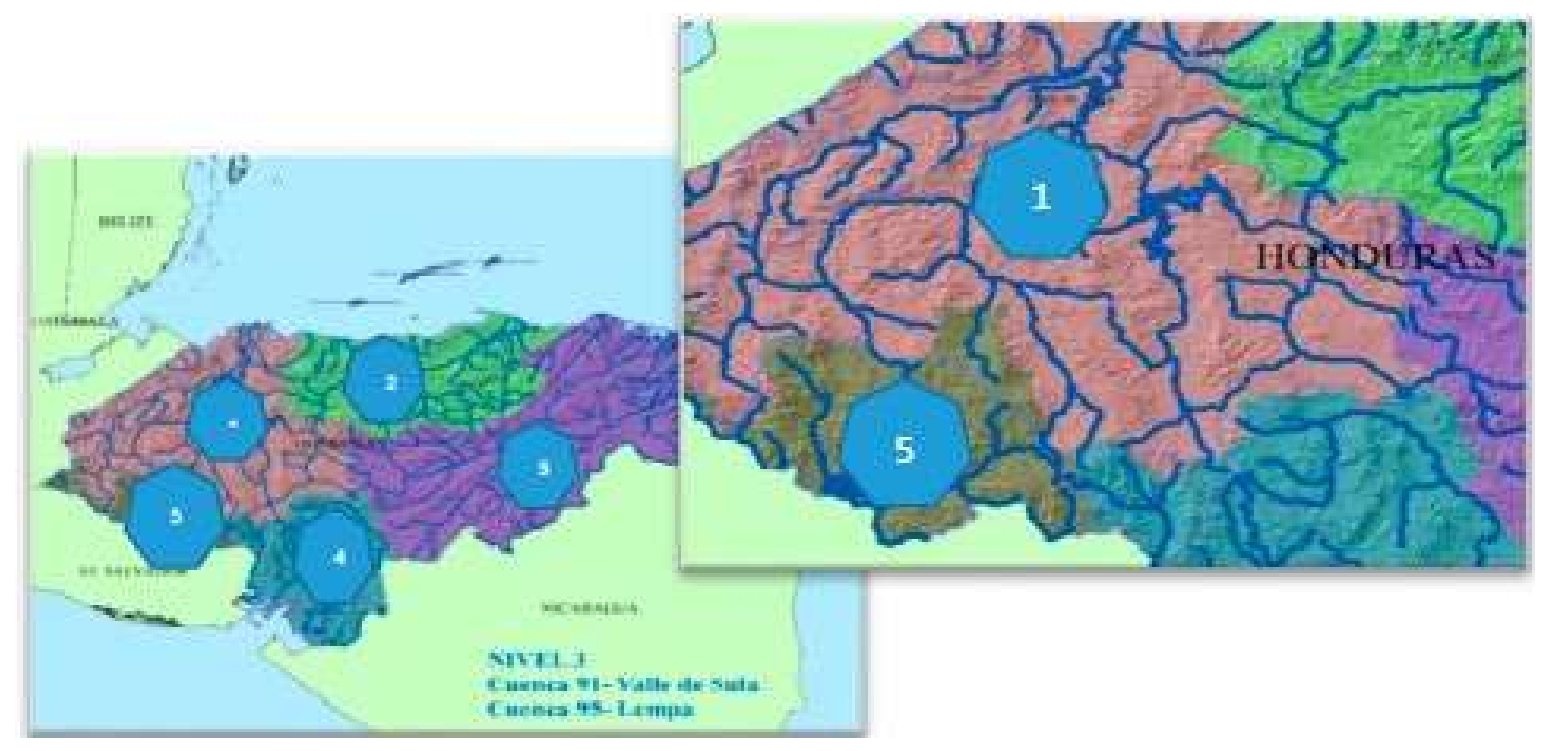

Figura 8. Delimitación de cuencas según Plan de Nación y Visión de País.

(Fuente: Elaboración propia).

Nivel 4, se utilizan la capa de Subcuencas a nivel nacional, aquí se ven incluidas las Sub cuencas EL Venado, Sub cuenca Grande Otoro, Subcuenca Humuya Alto y Subcuenca Selguapa. (Ver Figura 8).

El proceso de codificación fue realizado gracias a la información proporcionada por el Servicio Geológico de Estados Unidos y la estrategia nacional de Cuencas con que cuenta el estado de Honduras.

La realización del siguiente proceso de delimitación de las cuencas según la metodología se basó en la utilización de técnicas de análisis espacial tipo raster las cuales consisten en la delimitación de los ríos principales, la selección de las cuatro redes de drenaje de mayor tamaño (por cálculos de acumulación de flujo) hasta la 
generación de cuencas en formato raster, lo que posteriormente se convierte en un vector.

La dirección de flujo está determinada por la dirección más empinada de descendencia de cada celda o pixel. Esta se calcula como: Cambio de valor de z/ distancia *100. Se utilizó además un modelo digital de elevación cuyos datos almacenados representan valores de altitud. (Ver Figura 9).

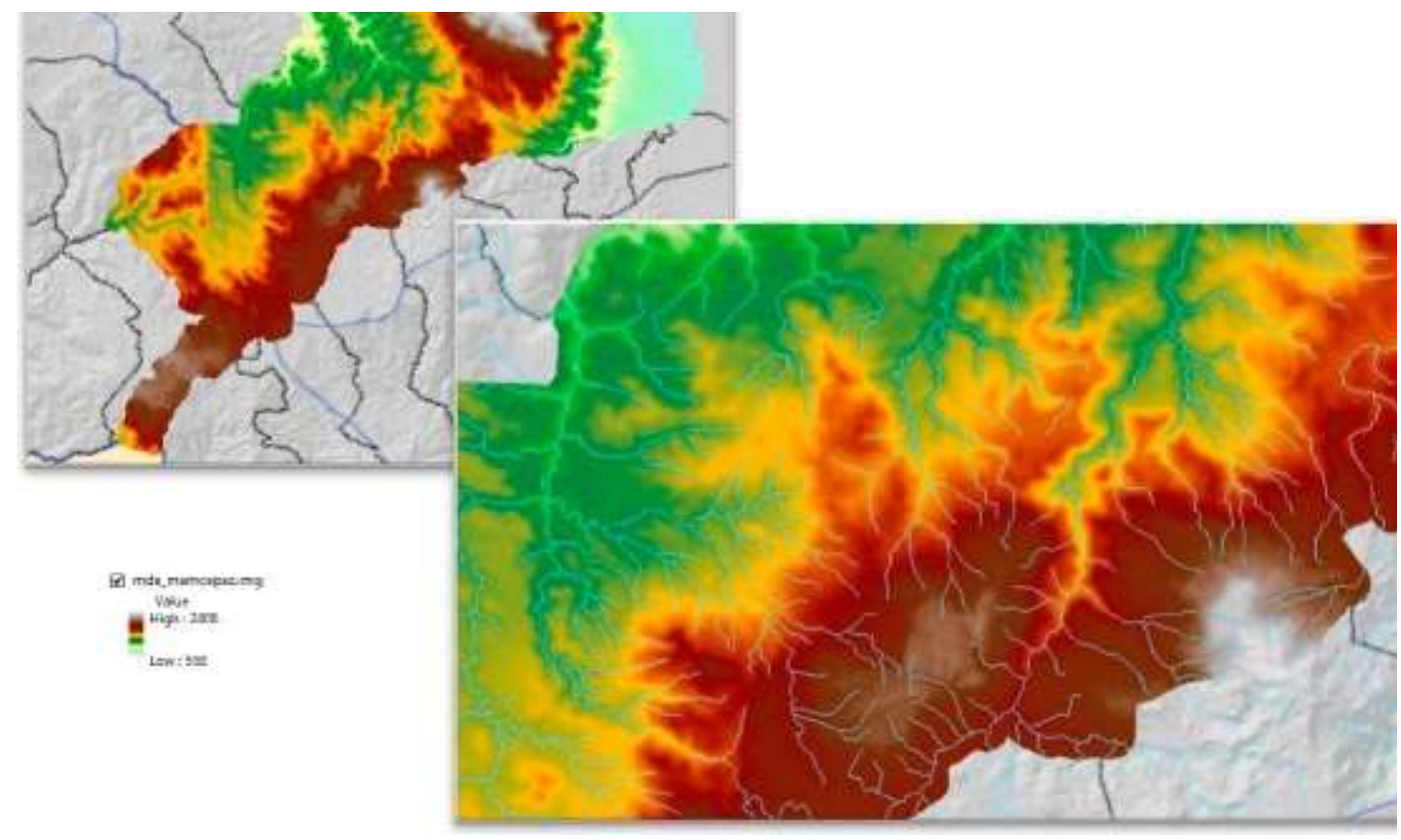

Figura 9. Modelo digital de Elevación de MAMCEPAZ. (Fuente: Elaboración propia).

Nivel 5 y 6 . El proceso de delimitación de las microcuencas se desarrolló mediante la utilización de herramientas con software especializado para la delimitación de microcuencas, convirtiendo las unidades hidrográficas al formato vectorial. (Ver Figura 10). 


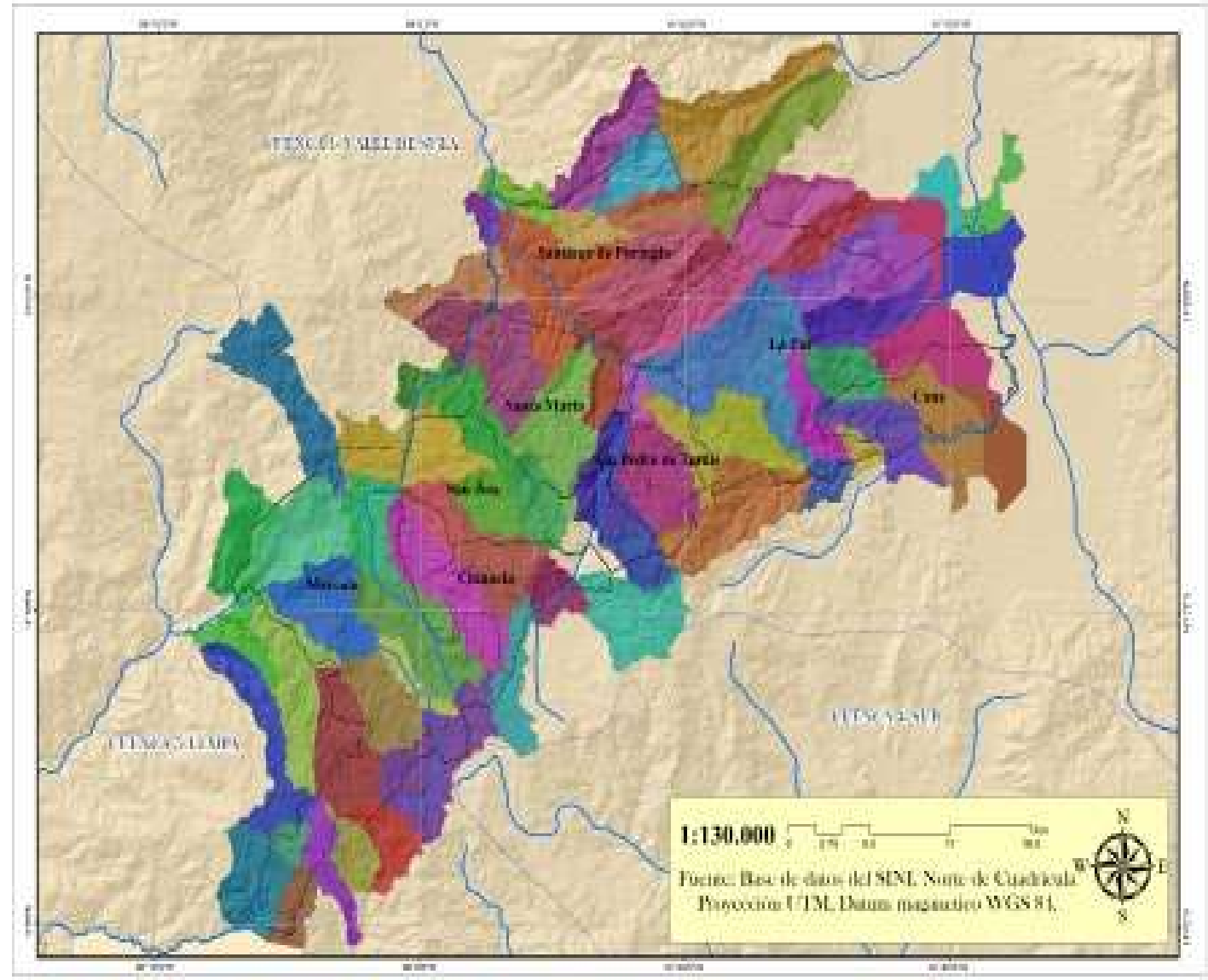

Figura 10. Delimitación de microcuencas MAMCEPAZ.

(Fuente: Elaboración propia).

Es de subrayar que estas microcuencas no comprenden únicamente el límite territorial de cada municipio, conociendo que una microcuenca tiene una unidad administrativa diferente de acuerdo con la dirección de flujo de agua. Como resultado se obtuvieron 77 microcuencas, las que se describen en los niveles 5 y 6 de acuerdo con las características de drenaje, quedando distribuidas de la siguiente manera (Ver tabla 2): 
Tabla 2. Distribución de Cuencas y Numero de Microcuencas de MAMCEPAZ.

\begin{tabular}{|c|c|c|}
\hline \multicolumn{3}{|l|}{ Nivel 5} \\
\hline Cuenca & Rio & Numero de Microcuencas \\
\hline \multirow[t]{2}{*}{ Valle de Sula } & Grande de Otoro & 9 \\
\hline & Humuya Alto & 22 \\
\hline Lempa & Selguapa & 11 \\
\hline \multicolumn{3}{|l|}{ Nivel 6} \\
\hline Cuenca & Rio & Numero de Microcuencas \\
\hline Valle de Sula & Grande & 27 \\
\hline Lempa & El Venado & 6 \\
\hline
\end{tabular}

(Fuente: Elaboración propia.)

\section{RESULTADOS}

Seguidamente convirtiendo las unidades hidrográficas al formato vectorial, generando polígonos en donde posteriormente se les asigna un valor de acuerdo con el nivel en que se encuentra. (Ver Figuras 11 y 12). 

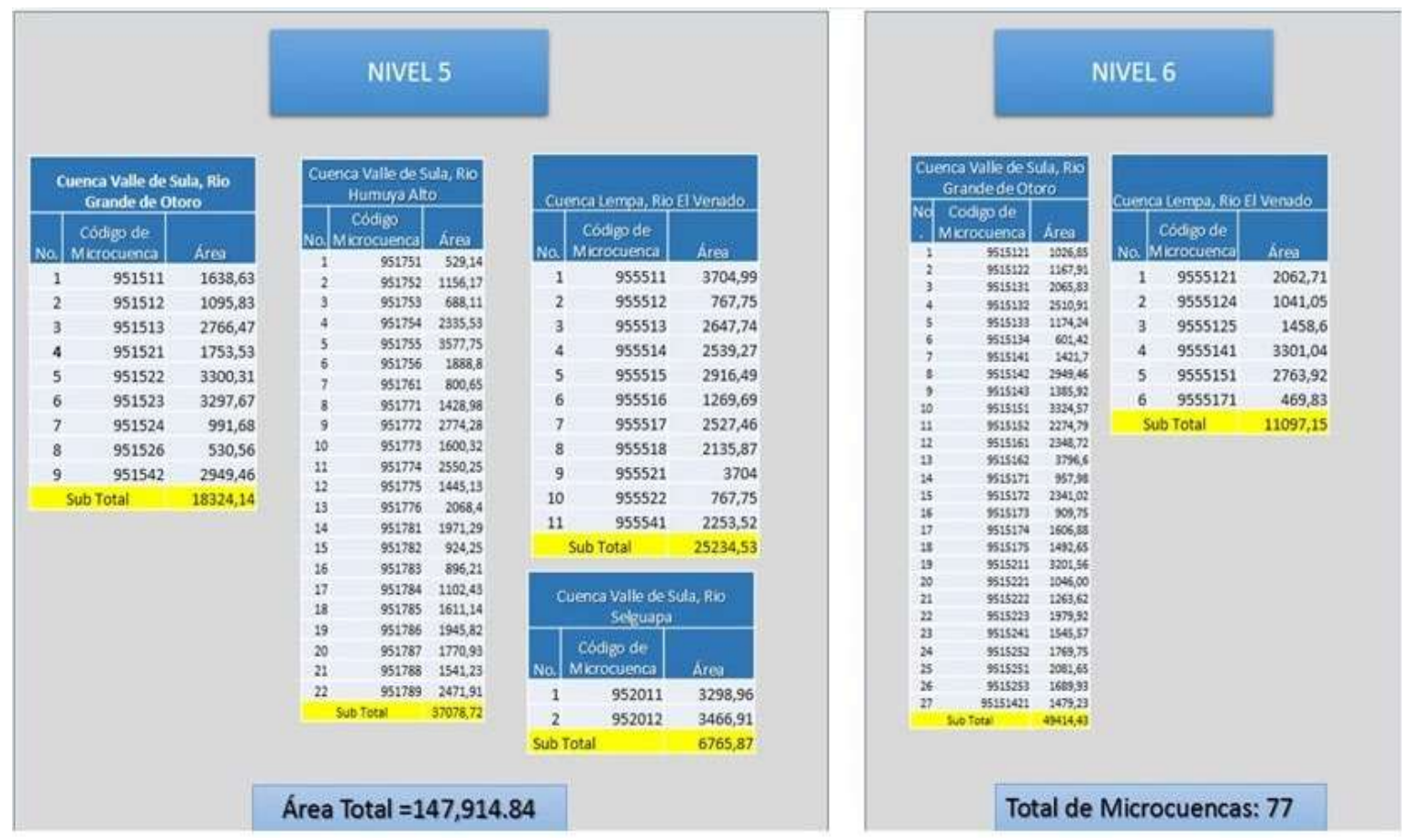

Figura 11. Asignación de Códigos en los diferentes Niveles de las Microcuencas de MAMCEPAZ. (Fuente: Elaboración propia).

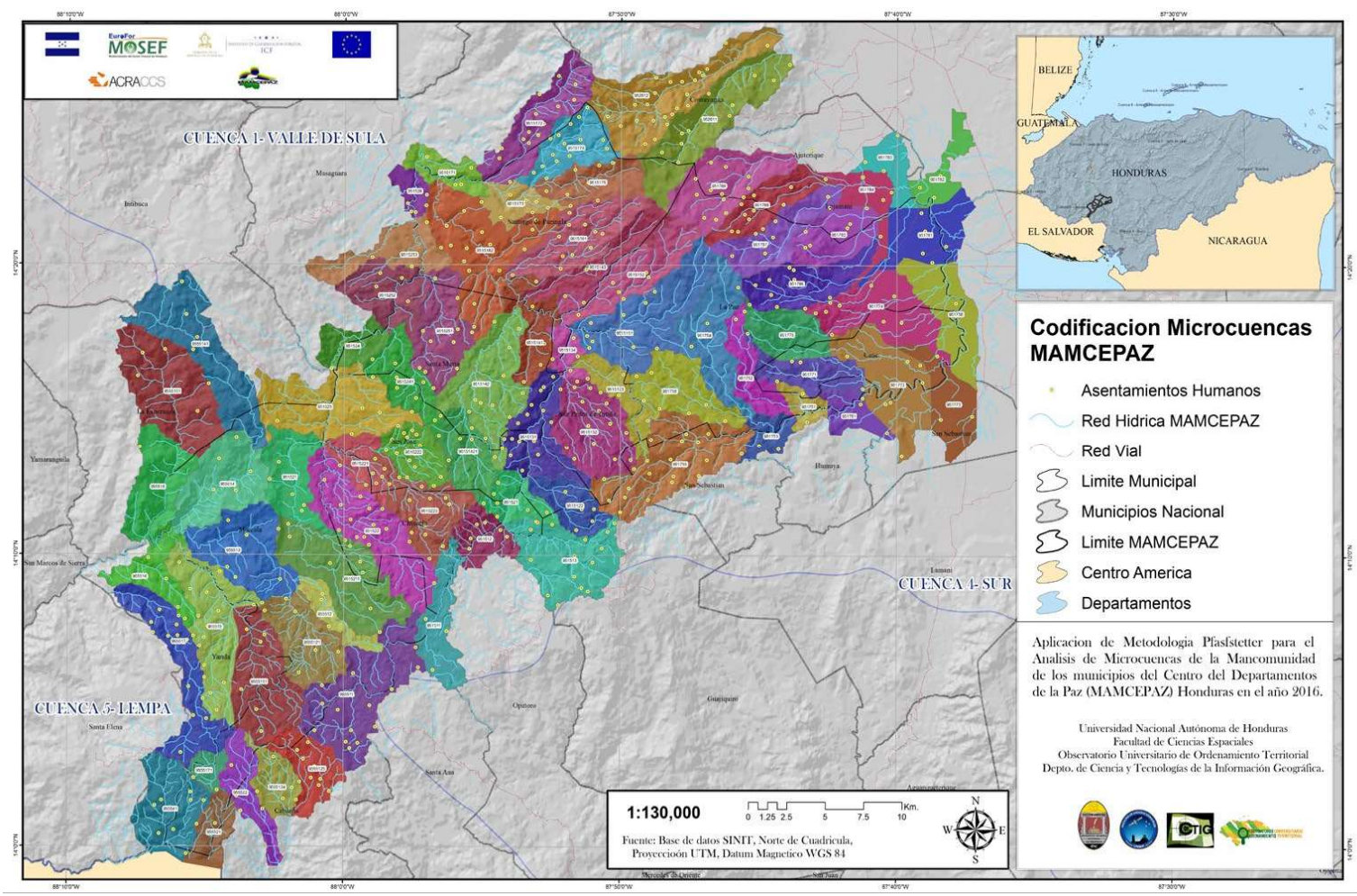

Figura 12. Mapa Final de Codificación de Microcuencas.

(Fuente: Elaboración propia). 


\section{Fase III. Validación de Campo}

Esta fase consistió en la inserción de proyectos de Interés Social y Ambiental dentro de las 77 Microcuencas, para ello se requirieron los 8 Planes Municipales de Ordenamiento Territorial (PMOT) en donde se enuncia la cartera de proyectos para cada asentamiento humano, esto a su vez enlazándolos con las microcuencas codificadas para tener un panorama de que proyecto corresponde a cada microcuenca y comunidad de acuerdo con sus necesidades. (ver Figura 13).

Una vez definidas las microcuencas de la Mancomunidad MAMCEPAZ, se realizó una ficha descriptiva para cada microcuenca identificada, en la que se detallan los datos en la ficha modelo.

Las fichas se establecen entonces como el elemento identificador de las características, problemas territoriales, presión poblacional y tamaño de cada microcuenca, además se identifica en las mismas los proyectos de inversión que coinciden con este espacio geográfico que fueron propuestos en cada Plan Municipal de Ordenamiento Territorial de MAMCEPAZ.

Se realizaron 77 fichas, que corresponden con la cantidad de microcuencas identificadas, las cuales se presentan en el acápite de anexos divididas por municipio.

\section{Fase IV. Identificación de Condiciones Biofísicas}

4.1 Reconocimiento de nombres de las microcuencas desarrolladas mediante metodología Pfafstetter, que consistió en la identificación del nombre de cada microcuenca mediante la identificación de la fuente de agua más cercana o la identificación de un hito territorial para poder identificar el nombre según zonas conocidas por los pobladores y no únicamente con un código generado de manera cartográfica. (Ver Figura 13). 


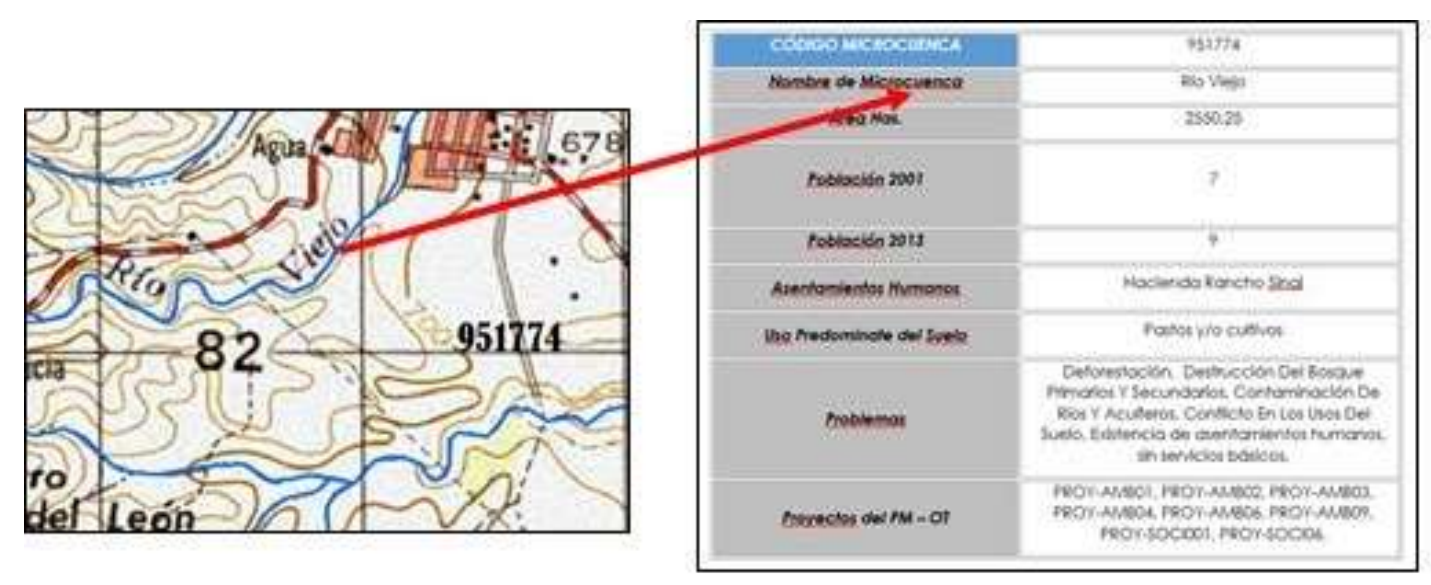

Figura 13. Proceso de Nombramiento de microcuencas.

(Fuente: Elaboración propia).

4.2 Uso Predominante del Suelo, en esta fase fue necesario la utilización de herramientas de geoprocesamiento como Intersect la cual calcula una intersección geométrica de las características de entrada. Las características o partes de las funciones que se superponen en todas las capas y / o clases de entidad se escribirán en la clase de entidad de salida y el Dissolve que agrupa las características basadas en atributos especificados (ESRI, 1995-2015), de esta manera y utilizando una base de datos de la última actualización de cobertura forestal de Honduras se analizó para determinar el uso de suelo predominante para cada microcuenca. (Ver Figura 14). 


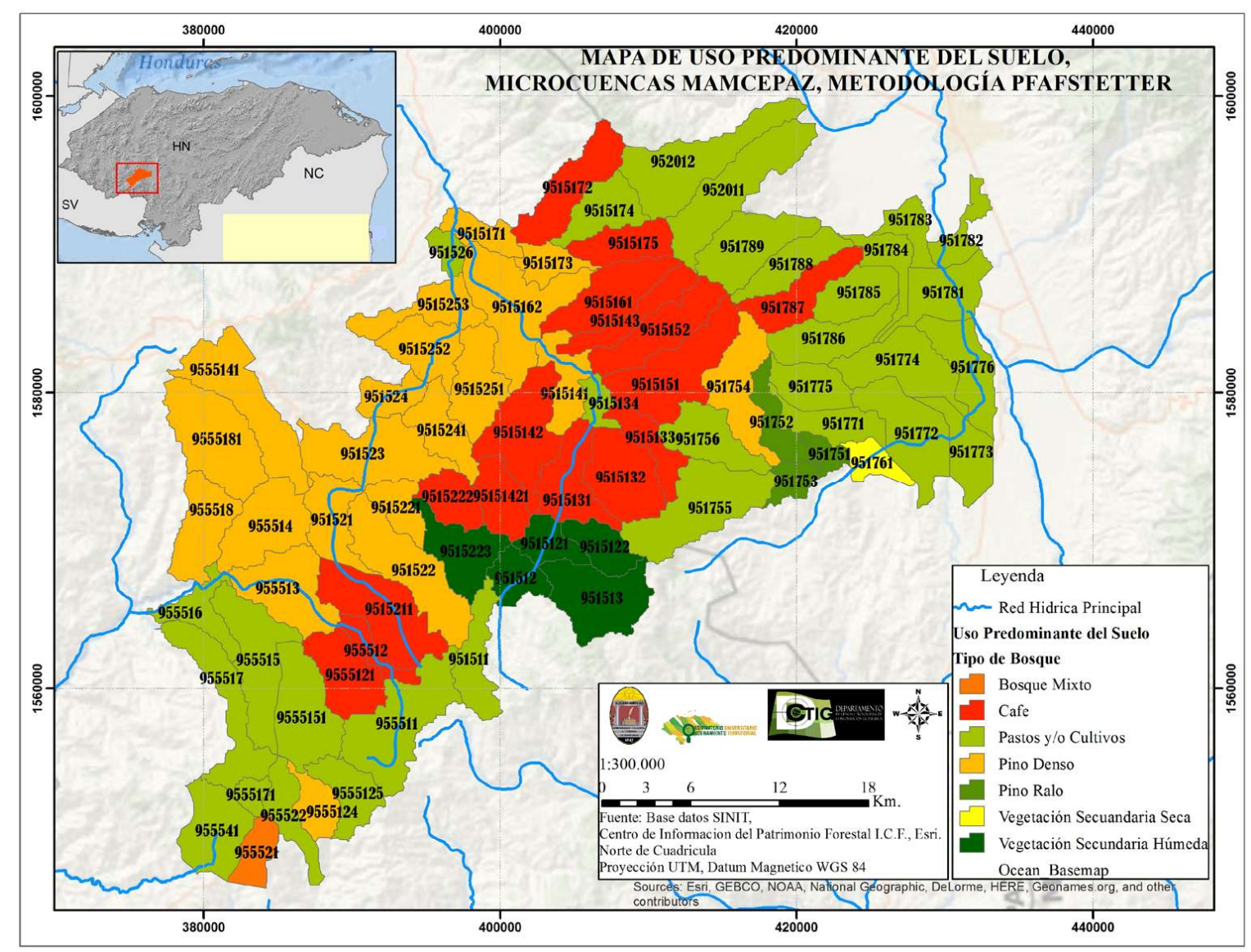

Figura 14. Mapa de Uso Predominante del Suelo.

(Fuente: Elaboración propia).

La Figura 14 muestra un gráfico donde se refleja la distribución de las áreas de acuerdo con los diferentes tipos de Uso Predominante encontrados en las Microcuencas de MAMCEPAZ, mostrándose que el uso que más predomina es el de Pastos y Cultivos con un $41 \%$, y el Pino Denso con un $28 \%$ es el tipo de bosque que más predomina en relación con el Bosque de Pino Ralo y Bosque Mixto. (Ver Figura 15) (Ver Tabla 3). 


\section{Area en Hectáreas}

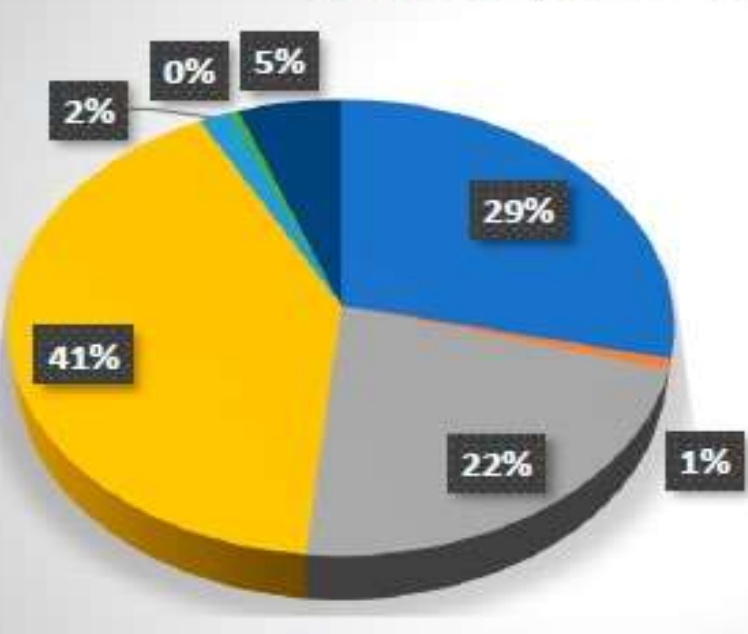

aino Denso

Bosque Mixto

Café

Pastos y/o Cultivos

Pino Ralo

Vegetación Secuandaria Seca

Figura 15. Distribución en áreas de los Usos de Suelo Predominantes en la MAMCEPAZ. (Fuente: Elaboración propia).

Tabla 3. Tipo de Bosque de acuerdo con el Uso de Suelo Predominante de MAMCEPAZ.

\begin{tabular}{|r|l|r|}
\hline \multicolumn{1}{|c|}{ No. Tipo de Bosque } & \multicolumn{1}{|c|}{ Area en Hectáreas } \\
\hline 1 & Pino Denso & 42007,37 \\
\hline 2 & Bosque Mixto & 986,70 \\
\hline 3 & Café & 32413,95 \\
\hline 4 & Pastos y/o Cullivos & 59595,65 \\
\hline 5 & Pino Ralo & 2373,43 \\
\hline 6 & Vegetación Secundaria Seca & 800,65 \\
\hline 7 & Vegetación Secundaria Húmeda & 8036,99 \\
\hline Total & & $146,214,75$ \\
\hline
\end{tabular}

(Fuente: Elaboración propia). 
4.3 Uso Recomendado del Suelo Se realizó un análisis combinado para determinar si existe subutilización o sobreutilización del uso del suelo según los usos del suelo recomendado.

La distribución de las áreas de acuerdo con los diferentes tipos de Uso recomendados encontrados en las Microcuencas de MAMCEPAZ, muestran que el uso recomendado más alto es el de apropiado para cultivos, seguida de Vocación Forestal. (Ver Figura 16) (Ver Tabla 4).

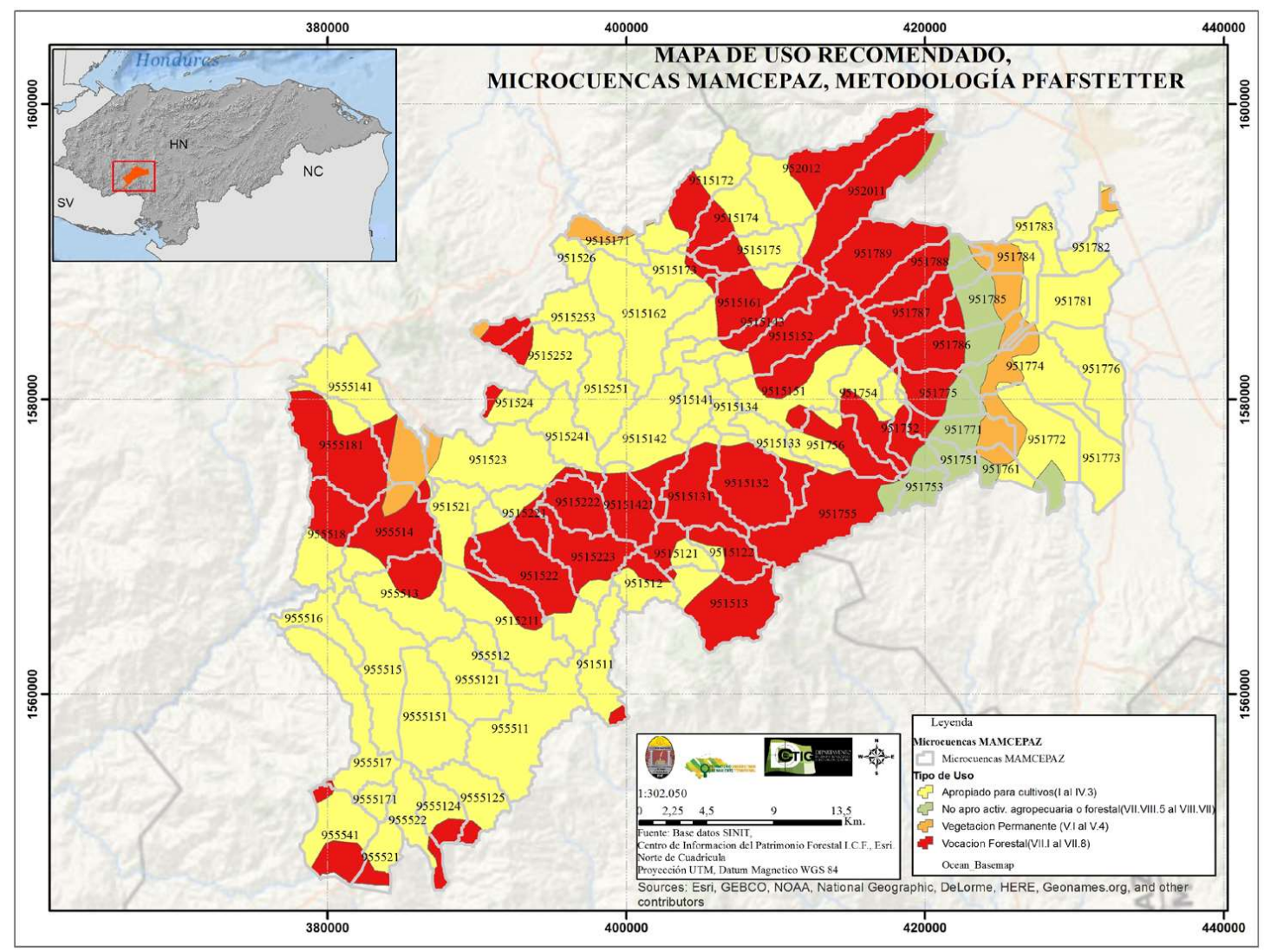

Figura 16. Mapa de Uso recomendado del suelo. (Fuente:Elaboración propia). 
Tabla 4. Áreas de Tipo de Bosque de acuerdo con el Uso de recomendado del suelo de MAMCEPAZ.

\begin{tabular}{|r|l|r|}
\hline No. & \multicolumn{1}{|c|}{ Tipo de Bosque } & \multicolumn{1}{c|}{ Area en Hectáreas } \\
\hline 1 & Apropiado para Cultivo & 79634.90 \\
\hline 2 & No apropiado para actividad agropecuaria o forestal & 6456.44 \\
\hline 3 & Vegetación Permanente & 5905.4 \\
\hline 4 & Vocación Forestal & 54218.01 \\
\hline Total & \multicolumn{1}{|l|}{} & $\mathbf{1 4 6 , 2 1 4 , 7 5}$ \\
\hline
\end{tabular}

(Fuente: Elaboración propia).

\section{FASE V. IDENTIFICACIÓN DE CONDICIONES SOCIOECONÓMICAS}

Carga Poblacional por Microcuencas

Para esta fase se actualizó la carga poblacional de cada microcuenca según los datos del censo de población y vivienda 2013. Además, se identificó el crecimiento poblacional analizando los datos de los censos de 1988, 2001 y 2013 para identificar las microcuencas con un mayor potencial de degradación debido a la ubicación de asentamientos humanos cercanos a fuentes de agua. (Ver figura 17). 
FICHAS MICROCUENCAS SAN PEDRO DE TUTULE|

\begin{tabular}{|c|c|}
\hline CODDIGO MICROCUENCA & 951755 \\
\hline Nombre de Microcuenca & San Miguel \\
\hline Área Has. & 3577.75 \\
\hline Población 2001 & 168 \\
\hline Población 2013 & 208 \\
\hline Asentamientos Humanos & Cerro de Upa, EL Carrizal \\
\hline Uso Predominante del Suelo & Pastos y/o Cultivos \\
\hline Problemas & $\begin{array}{c}\text { Deforestación, Destrucción Del Bosque } \\
\text { Primarios Y Secundarios, Incendios Forestales, } \\
\text { Contaminación De Ríos Y Acuiferos, Conflicto } \\
\text { En Los Usos Del Suelo, Deficiente manejo del } \\
\text { área protegida zona productora de agua El } \\
\text { Jilguero, Existencia de asentamientos } \\
\text { humanos, sin servicios básicos, }\end{array}$ \\
\hline Proyectos del PM - OT & $\begin{array}{l}\text { PROY-AMBO1, PROY-AMBO2, PROY-AMBO3, } \\
\text { PROY-AMBO5, PROY-AMBO6, PROY-AMBO9, } \\
\text { PROY-AMB10, PROY-AMB12, PROY-AMB14, }\end{array}$ \\
\hline
\end{tabular}

Figura 17. Ficha completa de microcuencas.

(Fuente: Elaboración Propia, datos utilizados de datos INE, (INE, 2013).

\section{CONCLUSIONES}

- Se realizó un análisis detallado de las microcuencas de la Mancomunidad MAMCEPAZ, resultados que se constituyen como una herramienta de gestión territorial para los 8 municipios pertenecientes a la Mancomunidad. La gestión territorial se constituye como una necesidad de primer orden para el adecuado uso del territorio influyendo directamente en la mejora de calidad de vida de la población. El análisis permitirá a los técnicos municipales y autoridades competentes conocer las necesidades, problemáticas y características territoriales de cada microcuenca.

- Se logró vincular los resultados de esta investigación con los Planes Municipales de Ordenamiento Territorial de MAMCEPAZ, inscritos ya en el RENOT, aspecto 
que facilitará a las autoridades municipales, monitorear y dar seguimiento al cumplimiento del subsistema ambiental de los PM-OT, constituyéndose esta investigación en un beneficio directo en la toma de decisiones técnicas en los 8 municipios de la mancomunidad.

- La presente investigación constituye un importante aporte en la transferencia de resultados de investigación y vinculación de la UNAH con diferentes sectores del país (en este caso 8 municipalidades categoría $C$ y $D$ según la categorización municipal del país) línea prioritaria de la DICYP y la UNAH en los 10 años de la reforma en investigación.

- Es importante destacar el incremento poblacional al que se han visto expuestas las microcuencas de la MAMCEPAZ entre el periodo 2001 -2013, lo que genera en la mayoría de los casos un conflicto en el uso del suelo, al no tener gran parte de las microcuencas la capacidad de acogida suficiente de la población. Se identifica el problema de la dispersión urbana en la mancomunidad, aspecto que influye de manera directa en el impacto ambiental del territorio.

\section{RECOMENDACIONES}

- Se recomienda ampliar los espacios de socialización de resultados dentro de las municipalidades y de la mancomunidad, con la finalidad de que la herramienta pueda colaborar en la gestión territorial de otros subsistemas como el urbano, infraestructuras y socioeconómico.

- Será necesario a lo interno de los municipios en base a los resultados de la investigación, generar planes operativos anuales para destinar presupuesto a la gestión adecuada de las microcuencas, siguiendo los lineamientos de los PM-OT y de los hallazgos de conflictos de usos del suelo y problemática para cada microcuenca.

- Se recomienda a las autoridades de la MAMCEPAZ crear una UTI (Unidad Técnica Intermunicipal) que permitirá dar seguimiento y soporte técnico a los técnicos UMAS de las 8 municipalidades para la gestión e implementación de estas herramientas de planificación. Las mancomunidades al ser entes técnicos más no políticos favorecen la implementación de estos instrumentos al contar con una mayor estabilidad laboral de sus técnicos y autoridades al trascender los 4 años de gobierno. 


\section{AGRADECIMIENTO}

A la Mancomunidad de Municipios del Centro de la Paz (MAMCEPAZ), a la ONG's ACRACCS , Modernización del Sector Forestal de Honduras (MOSEF), por su valiosa colaboración para el desarrollo de esta investigación.

\section{BIBLIOGRAFÍA}

- Antúnez, N. C. (2011). CRITERIOS TÉCNICOS DE DELIMITACIÓN Y CODIFICACION DE CUENCAS, CASO ESPECÍFICO REGIÓN ORIENTAL DEL PARAGUAY. Paraguay.

- ESRI. (1995-2015). ArcGis Resources. Obtenido de http://resources.arcgis. com/EN/HELP/MAIN/10.2/index.htm/\#//00170000005n000000.

- ICF, I. N. (2010). Estrategia Nacional de Manejo de Cuencas en Honduras. Tegucigalpa, Honduras.

- INE, I. N. (2013). Censo Población y Vivienda. Tegucigalpa, Honduras.

- INEGI-INE-CONAGUA. (2007). Documento técnico del mapa de Cuencas hidrográficas de México (escala 1: 250 000). México.

- INETER, A. U.-P. (2014). Cuencas Hidrográficas de Nicaragua bajo la metodología Pfafstetter. Nicaragua.

- INSTITUTO NACIONAL DE RECURSOS NATURALES, I. D. (2005). MARCO CONCEPTUAL Y MANEJO DE CUENCAS, EN EL PERU. Perú.

- SINIT, Sistema Nacional de Información Territorial de Honduras.

- SUR, U. (2008). Manual de procedimientos de Delimitación y Codificación de Unidad Hidrográfica. Colombia.

- Survey, U D.-U (2016). http://hydrosheds.cr.usgs.gov/datadownload.php. Obtenido de USGS-Science for a changing world:http://hidrosheds.cr.usgs.gov/ datadownload.php?repdata=3dem. 